The Astrophysical Journal, 549:274-280, 2001 March 1

(C) 2001. The American Astronomical Society. All rights reserved. Printed in U.S.A.

\title{
47 TUCANAE: THE SPECTROSCOPIC VERSUS COLOR-MAGNITUDE DIAGRAM AGE DISCREPANCY
}

\author{
Alexandre Vazdekis, ${ }^{1}$ Maurizio Salaris, ${ }^{2}$ Nobuo Arimoto, ${ }^{3}$ and James A. Rose ${ }^{4}$ \\ Received 2000 July 31; accepted 2000 November 2
}

\begin{abstract}
We investigate current problems in obtaining reliable ages for old stellar systems based on stellar population synthesis modeling of their integrated spectra. In particular, we address the large ages derived for the globular cluster 47 Tuc, which is at odds with its color-magnitude diagram (CMD) age. Using a new age indicator, $\mathrm{H} \gamma_{\sigma<130}$, which is particularly effective at breaking the degeneracy between age and metallicity, we confirm the discrepancy between the spectroscopic age and the CMD age of 47 Tuc, in that the spectroscopic age is much older. Nebular emission appears unlikely to be a source for weakening the observed Balmer lines. We then explore a number of key parameters affecting the temperature of turnoff stars, which are the main contributors to the Balmer lines for old metal-rich stellar populations. We find that $\alpha$-enhanced isochrones with atomic diffusion included not only provide a good fit to the CMD of 47 Tuc but also lead to a spectroscopic age in better agreement with the CMD age.

Subject headings: galaxies: evolution - galaxies: stellar content - globular clusters: general globular clusters: individual (47 Tucanae) - stars: evolution
\end{abstract}

\section{INTRODUCTION}

An estimate of the mean luminosity-weighted stellar age of an early-type galaxy represents a major step in unveiling its true star formation history. However, to derive reliable information about stellar ages from the integrated light of unresolved galaxies one must deal with the age-metallicity degeneracy problem, which affects not only integrated colors but also absorption-line strengths (Worthey 1994). Recently, new age-dating techniques based on the Balmer lines (e.g., Jones \& Worthey 1995; Vazdekis \& Arimoto 1999, hereafter VA99) have shown great promise in untangling the age-metallicity degeneracy. These techniques should be tested and calibrated on the metal-rich Galactic globular clusters (GCs) for which, unlike elliptical galaxies, independent age estimates are possible (Gibson et al. 1999, hereafter G99) by means of the color-magnitude diagram (CMD) of their resolved stellar population.

The application of the new age-dating techniques to very high signal-to-noise ratio $(\mathrm{S} / \mathrm{N})$ spectra of metal-rich Galactic GCs has revealed two major concerns: (1) the obtained ages are unreasonably large (greater than $20 \mathrm{Gyr}$; Jones 1999; Cohen, Blakeslee, \& Ryzhov 1998; G99; VA99), and (2) a severe disagreement is found between the spectroscopic and CMD ages of 47 Tuc (G99). The CMD-derived ages may be sensitive to the dating method employed (e.g., Alonso et al. 1997) and to the input physics of the theoretical isochrones used as a reference (e.g., Salaris \& Weiss 1998, hereafter SW98). However, a variety of recent CMDbased age determinations for 47 Tuc have consistently found its age to lie within 9-12.5 Gyr (SW98; Gratton et al. 1997; Carretta et al. 2000; Liu \& Chaboyer 2000).

\footnotetext{
${ }^{1}$ Department of Physics, University of Durham, Durham DH1 3LE, England, UK; Alexandre.Vazdekis@durham.ac.uk. Present address: Instituto de Astrofisica de Canarias, E-38200 La Laguna, Tenerife, Spain; vazdekis@1l.iac.es.

${ }^{2}$ Liverpool John Moores University, Twelve Quays House, Egerton Wharf, Birkenhead CH41 1LD, England, UK; ms@astro.livjm.ac.uk.

${ }^{3}$ Institute of Astronomy, University of Tokyo, Osawa 2-21-1, Mitaka, Tokyo 181-0015, Japan; arimoto@mtk.ioa.s.u-tokyo.ac.jp.

4 Department of Physics and Astronomy, University of North Carolina, Chapel Hill, NC 27599; jim@physics.unc.edu.
}

These CMD-derived ages are substantially younger than the greater than $20 \mathrm{Gyr}$ spectroscopically derived age obtained by G99 using the age indicator of Jones \& Worthey (1995) and Worthey (1994) stellar population models. This discrepancy shows clearly that current stellar population synthesis models used for interpreting the integrated light of stellar systems may have severe zero-point problems.

In $\S 2$ we improve the spectroscopic age-dating technique which confirms a large spectroscopic age for 47 Tuc. In $\S 3$ we discuss the possible origin of the problem, explore several theoretical parameters, and suggest a possible solution. Finally, in $\S 4$ we present our conclusions.

\section{A NEW H $\gamma$ AGE INDICATOR: 47 TUCANAE AGE ESTIMATE}

In this study we make use of the evolutionary stellar population synthesis model of Vazdekis (1999, hereafter V99), which predicts spectral energy distributions in the optical wavelength range for single-burst old-aged stellar populations (SSPs) of metallicities $-0.7 \leq$ $\log \left(Z / Z_{\odot}\right) \leq+0.2$, at resolution $1.8 \AA$ (FWHM). This approach is different from the one followed by previous models (e.g., Worthey 1994; Vazdekis et al. 1996) which used mostly the Lick/IDS polynomial fitting functions (Worthey et al. 1994; Worthey \& Ottaviani 1997) to relate the strengths of selected absorption features to stellar atmospheric parameters. These fitting functions are based on the Lick/IDS stellar library (FWHM $\sim 9 \AA$; Worthey et al. 1994), thus limiting the sensitivity of the models to weak features. However, V99 model provides full spectral energy distributions (SEDs) rather than predicted index strengths, and therefore the Lick indices defined in Worthey et al. (1994) and Worthey \& Ottaviani (1997), as well as those of Rose (1994) and Jones \& Worthey (1995), are measured directly on the SEDs of V99 models (without the use of any fitting functions). This approach makes it easy to define new indices and even to confront the model predictions to the detailed structure of observed absorption features. V99 models have been recently updated (A. Vazdekis 2001, in preparation) with Girardi et al. (2000) scaled-solar iso- 
chrones and new empirical photometric libraries, such as Alonso, Arribas, \& Martínez-Roger (1999).

The chief impediment to obtaining reliable ages of elliptical galaxies is caused by the degenerate effects of age and metallicity on the integrated spectra of old stellar populations (e.g., Worthey 1994). Recently, VA99 have proposed a new age indicator, centered on $\mathrm{H} \gamma$, which provides unprecedented power for breaking the age-metallicity degeneracy. Their index is a pseudo-equivalent-width measurement, relying on the pseudocontinuum peaks immediately longward and shortward of $\mathrm{H} \gamma$ and is an improvement over earlier pseudo-equivalent-width $\mathrm{H} \gamma$ indices (Rose 1994; Jones \& Worthey 1995) in being relatively insensitive to spectral resolution. We have redefined the VA99 index to take full advantage of the information available in an integrated spectrum with $\sigma \sim 100 \mathrm{~km} \mathrm{~s}^{-1}$ and to make the index as insensitive as possible to metallicity. This new index, $\mathrm{H} \gamma_{\sigma<130}$, is composed of two pseudocontinua at 4329.000-4340.468 and 4352.500-4368.250 and the feature at $4333.250-4363.000 \AA$. The main difference with respect to the VA99 definition is that the feature now also covers the neighboring metallic line centered on $\lambda \sim 4352 \AA$. The two pseudocontinua severely overlap the index passband aiming at making $\mathrm{H} \gamma_{\sigma<130}$ stable against changes in the spectral resolution (see below) and insensitive to metallicity variations on the basis of the compensating effect raised up by VA99: at a given age, $\mathrm{H} \gamma$ strengthens with metallicity owing to the adjacent metallic absorption, but on the other hand, the pseudocontinua are depressed by the effects of the neighboring $\mathrm{Fe} \mathrm{I}$ lines on both sides of $\mathrm{H} \gamma$ (see VA99 for an extensive explanation).

Figure 1 shows the age disentangling power of the $\mathrm{H} \gamma_{\sigma<130}$. In the left panel, the indices for both M32 (Jones 1999 spectrum) and for 47 Tuc (Rose 1994 spectrum) are plotted relative to the models. The age estimate for M32 is consistent with VA99 result ( $\sim 4 \mathrm{Gyr}$ ) based on the earlier $\mathrm{H} \gamma$ index. The principal issue for this paper, however, is the very large age inferred for 47 Tuc, i.e., well in excess of 15 Gyr. This disturbingly large inferred age confirms that already obtained by G99 and VA99 and thus is a feature of all recent studies of this cluster (based on different observational spectra). The right panel of Figure 1 shows the insensitivity of $\mathrm{H} \gamma_{\sigma<130}$ to resolution in the range $60 \mathrm{~km}$ $\mathrm{s}^{-1}<\sigma<130 \mathrm{~km} \mathrm{~s}^{-1}$. Spectra of $\mathrm{S} / \mathrm{N}(\AA) \sim 175$ and a very

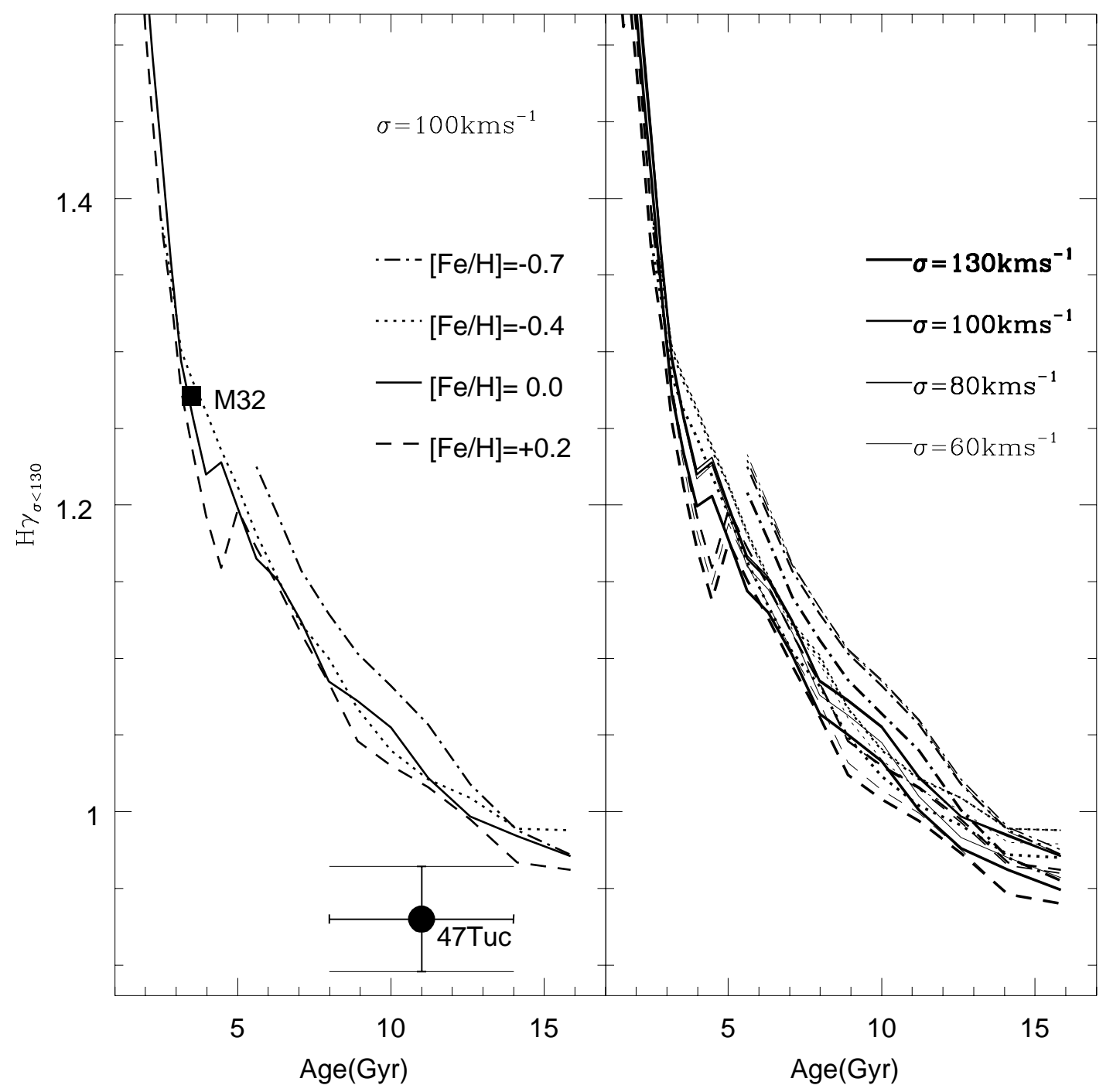

FIG. 1.-Left panel: New $\mathrm{H} \gamma_{\sigma<130}$ age indicator measured on V99 model spectral library smoothed to $\sigma \sim 100 \mathrm{~km} \mathrm{~s}^{-1}$ to match the resolution of the very high S/N spectrum of 47 Tuc (Rose 1994). The size of the error bar along the age axis represents the maximum range for the most recent CMD age estimates $(9 \pm 1$ Gyr in SW98 and $12.5 \pm 1.5 \mathrm{Gyr}$ in Liu \& Chaboyer 2000), and therefore we plotted 47 Tuc value at 11 Gyr. Right panel: Test for the stability of $\mathrm{H} \gamma_{\sigma<130}$ against resolution in the range $60 \mathrm{~km} \mathrm{~s}^{-1}<\sigma<130 \mathrm{~km} \mathrm{~s}^{-1}$. 
careful correction of any wavelength shift (see VA99) are required to take the full advantage of this index.

Figure 2 further illustrates the age-metallicity resolving power achieved with the $\mathrm{H} \gamma_{\sigma<130}$ index and further clarifies the troublesome problem of the inferred age for 47 Tuc. Here $\mathrm{H} \gamma_{\sigma<130}$ is plotted versus several different indices defined in Worthey et al. (1994) and Rose (1994). All the indices were measured directly on the model SEDs of V99 smoothed to match the resolution of the spectrum of 47 Tuc. Again, all index plots indicate an age for 47 Tuc in excess of 15 Gyr. In addition, most of the plots in Figure 2 suggest a metallicity for the cluster around 0.1-0.2 dex lower than the Carretta \& Gratton (1997) value of [Fe/ $\mathrm{H}]=-0.7$, which is based on high-dispersion spectra of individual red giants. On the other hand, Gratton \& Sneden (1991) and Brown \& Wallerstein (1992) obtained [Fe/ $\mathrm{H}] \sim-0.8$ and -0.9 , respectively. Recently, Liu \& Chaboyer (2000) obtained $[\mathrm{Fe} / \mathrm{H}]=-0.95$ using the isochrone fitting technique. However, it bears emphasizing that the uncertainty in metallicity does not affect the age discrepancy shown here.

\section{DISCUSSION}

In this section we explore possible causes for the 47 Tuc age discrepancy: emission-line contamination, possible peculiarity of the cluster, horizontal-branch contribution, mixing length calibration, $\alpha$-element enhancement, initial helium abundance, and atomic diffusion.

\subsection{Nebular Emission}

G99 pointed out that gas emission could be partially filling in $\mathrm{H} \gamma$. We therefore studied other Balmer lines, since emission would affect them by differing amounts, according to standard case B recombination line physics (e.g., Osterbrock 1989). Figure 3 shows that the derived age for 47 Tuc is essentially independent of the Balmer line used. If emission fill-in were the cause of the large spectroscopic age, one would expect the ages derived from $\mathrm{H} \beta$ and $\mathrm{H} \delta$ to be older and younger, respectively, than that obtained from $\mathrm{H} \gamma$. To quantify this statement, we have used a fluxcalibrated spectrum of the Orion Nebula as a template for a case $\mathrm{B}$ hydrogen recombination spectrum. In fact, we adjusted the Balmer line intensities to exactly match the case B prescription. We then normalized the Orion spectrum so that when subtracted from the 47 Tuc spectrum at $\mathrm{H} \beta$, the derived age from the $\mathrm{H} \beta$ index is reduced to $11 \mathrm{Gyr}$. The ages derived from the $\mathrm{H} \gamma_{\sigma<130}$ and $\mathrm{H} \delta_{F}$ indices are $\sim 12.5$ Gyr and $\sim 14$ Gyr. Thus, the emission-corrected ages from different Balmer lines are, in fact, discordant, although the degree of discrepancy is probably not sufficient to eliminate the emission hypothesis altogether.

An additional problem for the emission hypothesis comes from the fact that spectra of other metal-rich Galactic globular clusters exhibit the same "anomalous" behavior in $\mathrm{H} \gamma$ as 47 Tuc. Specifically, in Rose (1994) a spectrum formed from the composite of four metal-rich globulars (NGC 6356, NGC 6624, M69, and M71) is compared to that of 47 Tuc (and M32) and shown to be nearly indistinguishable from 47 Tuc in all respects, including the pseudoequivalent-width index for $\mathrm{H} \gamma$. We have further quantified this fact by measuring the $\mathrm{H} \gamma_{\sigma<130}$ indices for the four clusters. On average, $\mathrm{H} \gamma_{\sigma<130}$ for the four clusters is 0.87 , i.e., slightly lower even than for 47 Tuc. Thus, if the emission hypothesis is correct, then in composite, the other four clusters must have essentially the same amount of emission fill-in as 47 Tuc. Such a scenario appears to be rather con-
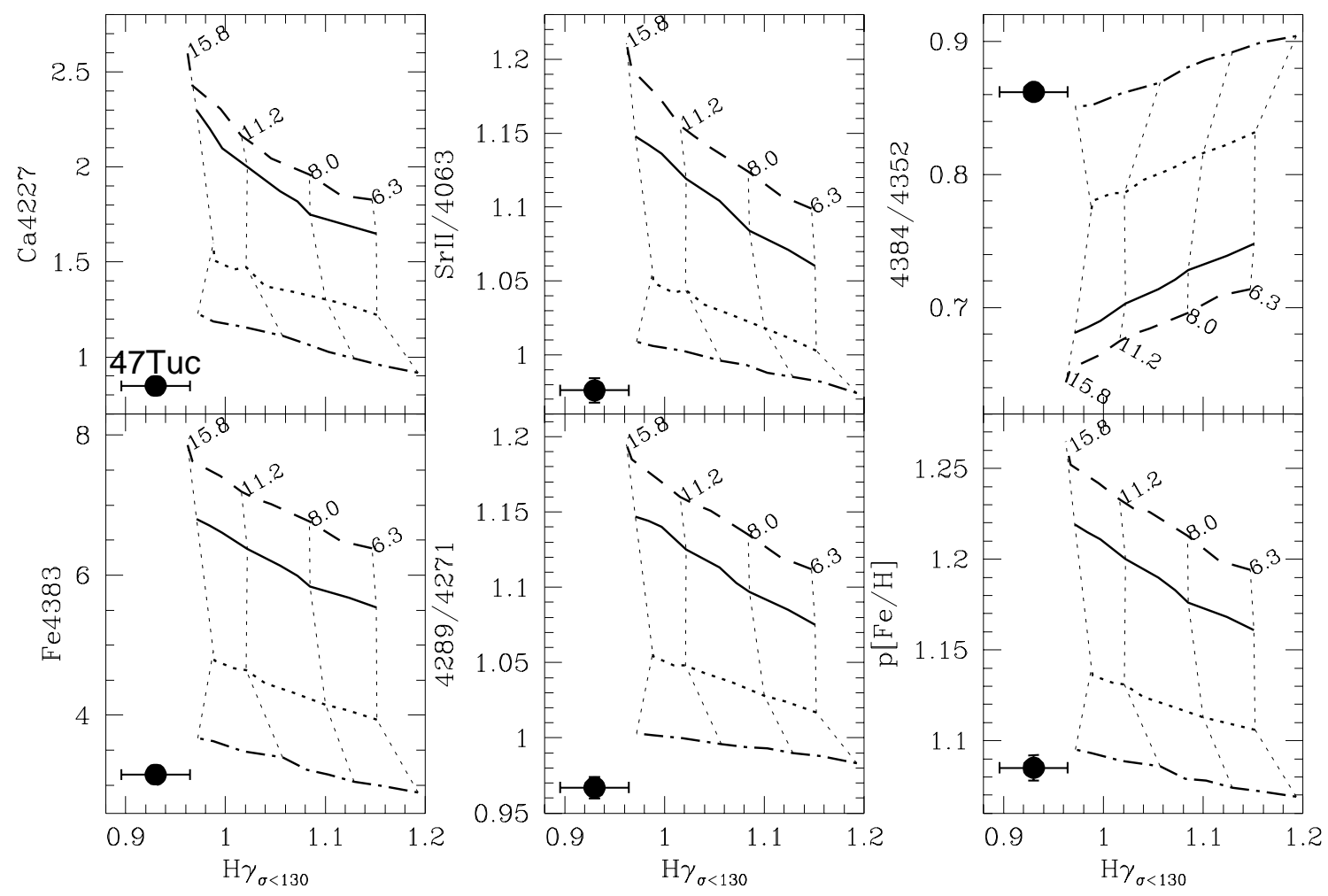

FIG. 2. - $\mathrm{H} \gamma_{\sigma<130}$ vs. different indices as defined in Worthey et al. (1994) (Ca4227; Fe4383) and Rose (1994). All of these indices were measured on the 47 Tuc spectrum of Rose (1994) and V99 models, smoothed to $\sigma=100 \mathrm{~km} \mathrm{~s}^{-1}$. Line types as in Fig. 1, while thin dotted lines mean models of equal ages indicated by the numbers (Gyr). 

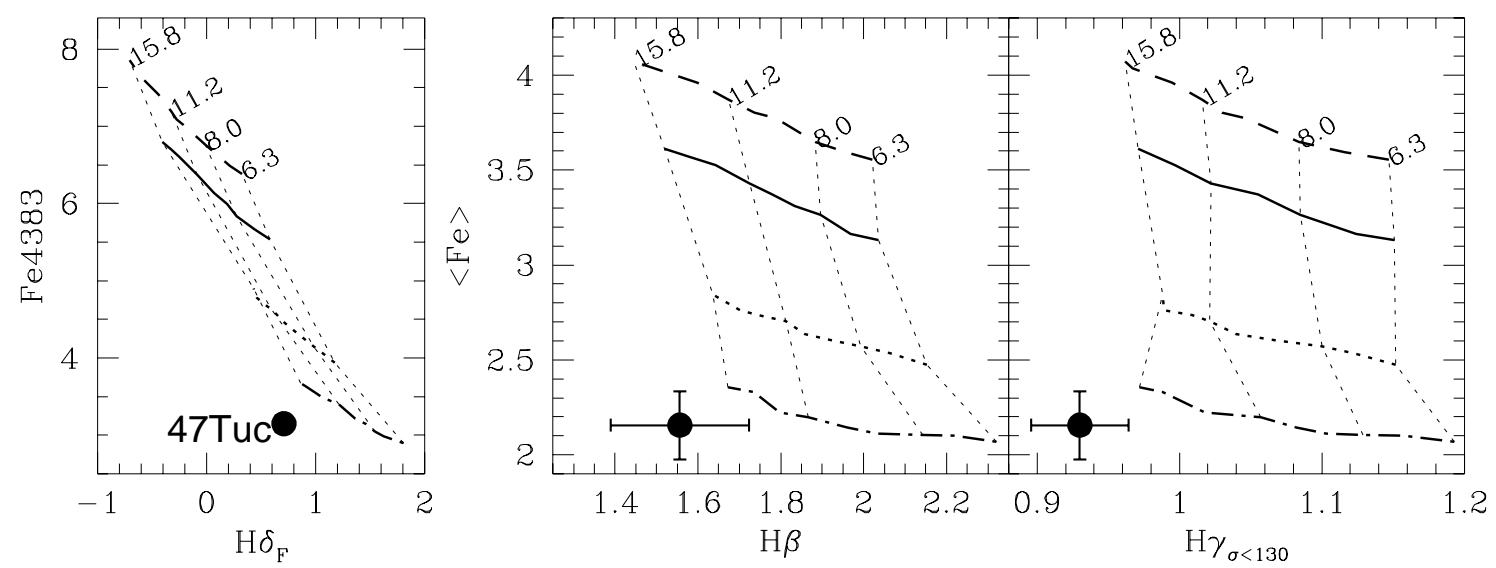

Fig. 3. - Left: $\mathrm{H} \delta_{F}$ as defined in Worthey \& Ottaviani (1997) is plotted vs. Fe4383. Middle: $\mathrm{H} \beta$ vs. $\langle\mathrm{Fe}\rangle$ [i.e., $\left.\frac{1}{2}(\mathrm{Fe} 5270+\mathrm{Fe} 5335)\right]$ (index definitions from Worthey et al. 1994). Since $\mathrm{H} \beta$ is not covered by the spectral range of the spectrum of Rose (1994), we used the spectrum of Covino, Galletti, \& Pasinetti (1995) with FWHM = 3.4 $\AA$. V99 models were smoothed to match this resolution. Right: $\mathrm{H} \gamma_{\sigma<130}$ vs. $\langle\mathrm{Fe}\rangle\left(\mathrm{H} \gamma_{\sigma<130}\right.$ measured on the Rose 1994 spectrum).

trived. In short, emission fill-in appears to be an unlikely source of the weak Balmer lines in 47 Tuc, but a more definitive assessment of the emission hypothesis could be achieved via deep interference filter imaging of the cluster.

In mentioning the other four metal-rich globular clusters it is also worth noting that there are still some integrated light properties of metal-rich clusters (e.g., anticorrelation between strengths of $\mathrm{CN}$ bands and the Sr II $\lambda 4077$ line) that remain unexplained among both Galactic (Rose \& Tripicco 1986) and M31 globular clusters (Burstein et al. 1984; Tripicco 1989).

\subsection{Is 47 Tucanae a Peculiar Cluster?}

A second possibility is that 47 Tuc is peculiar among Galactic GCs. However, by sketching the models of Vazdekis et al. (1996) to the $\mathrm{Mg}_{2}-\mathrm{H} \beta$ plot of Burstein et al. (1984; cf. their Fig. $5 k$ ) we see that a number of metal-rich Milky Way GCs fall well below the model lines (e.g., models of $[\mathrm{Fe} / \mathrm{H}]=-0.7$ and $16 \mathrm{Gyr}$ yield $\mathrm{Mg}_{2} \sim 0.17$ and $\mathrm{H} \beta \sim 1.7$ ). The same result is achieved if Worthey (1994) model grids are used. VA99, Vazdekis et al. (1996; cf. their Fig. 8), and Cohen et al. (1998) find analogous results for a set of metal-rich Galactic GCs. Finally, as mentioned above, the composite spectrum of four metal-rich clusters analyzed in Rose (1994) is similar in all respects to 47 Tuc.

\subsection{The Horizontal-Branch Contribution}

The Balmer indices are dominated by the hottest stars along the isochrone (e.g., Rose 1994; Worthey 1994; Buzzoni, Mantegazza \& Gariboldi 1994), i.e., by turnoff (TO) and horizontal-branch (HB) stars. For a GC with a red HB such as 47 Tuc we have verified that the contribution due to HB stars is negligible for this index. In fact, a decrease of the HB stars temperature by $150 \mathrm{~K}$ yields $\mathrm{H} \gamma_{\sigma<130}$ values smaller by less than $0.01 \AA$, in the range of ages 6-16 Gyr. Therefore, the discrepancy between the integrated light and CMD ages must be found in the TO. On the basis of Figure 1 and the TO temperatures of our isochrones, we estimate that to decrease $\mathrm{H} \gamma_{\sigma<130}$ by $\sim 0.050 \AA$ (to match the observed value for typical CMD ages) requires a TO cooler by $\sim 200 \mathrm{~K}$, for a given age. We therefore should look at those parameters affecting the TO effective temperature.

\subsection{Mixing Length}

The stellar models we use adopt a solar-calibrated value of the mixing length. This assumption is in agreement with results from current two-dimensional hydrodynamical simulation of superadiabatic stellar convection, even for non-solar-metallicity stars (Freytag \& Salaris 1999). However, we have also tested an alternative prescription for the treatment of superadiabatic convection in stellar envelopes, namely, the full spectrum turbulence theory (FST; see, e.g., D'Antona, Caloi, \& Mazzitelli 1997). We find that for the age and metallicity regime typical of 47 Tuc, the difference of TO effective temperatures between solarcalibrated mixing length and FST models is negligible $(\sim 10 \mathrm{~K})$.

\section{5. $\alpha$-Element Enhancement}

The chemical composition of 47 Tuc stars (and of Galactic GCs in general) is enhanced in $\alpha$-elements (we mean mainly $\mathrm{O}, \mathrm{Ne}, \mathrm{Mg}, \mathrm{Si}, \mathrm{S}, \mathrm{Ca}$, and $\mathrm{Ti}$; see, e.g., the review by Carney 1996). Spectroscopic determinations of $\alpha$-element abundances in 47 Tuc stars have been performed by Gratton, Quarta, \& Ortolani (1986); Brown, Wallerstein, \& Oke (1990); Brown \& Wallerstein (1992); and Norris \& Da Costa (1995). Their results, as summarized by Carney (1996), provide $\langle[\mathrm{O} / \mathrm{Fe}]\rangle=0.53 \pm 0.08$ and an average enhancement of $[\mathrm{Si}+\mathrm{Ca}+\mathrm{Ti} / \mathrm{Fe}]$ of about $0.20 \mathrm{dex}$.

Moreover, as demonstrated by SW98 and confirmed by Vandenberg, Swenson, \& Alexander (2000), for the $[\alpha / \mathrm{Fe}]$ ratios observed in Galactic GCs $([\alpha / \mathrm{Fe}] \sim 0.3-0.4)$ and $[\mathrm{Fe} / \mathrm{H}]>-1$, it is not valid to use scaled-solar isochrones with the same global metallicity as the $\alpha$-enhanced ones to approximate the effect of the $\alpha$-element enhancement. As a preliminary test we computed selected scaled-solar isochrones using the same code and input physics as in SW98, providing a good agreement for the TO temperatures with the corresponding isochrones of Girardi et al. (2000; used as scaled-solar ones in our population synthesis code). When comparing $\alpha$-enhanced with scaled-solar isochrones, the former have a hotter TO if the comparison is made at the same global metallicity $(Z=0.008)$, while if the comparison is made at the same $[\mathrm{Fe} / \mathrm{H}]=-0.7$, they have a cooler TO (see SW98).

We therefore implemented in our population synthesis code the $\alpha$-enhanced isochrones $(\langle[\alpha / \mathrm{Fe}]\rangle=0.4)$ of SW98, 
transformed to the observational plane following our empirical prescriptions (the same ones that we applied to the Girardi et al. 2000 isochrones). The referee asked us to state that we did not change the input empirical stellar spectral database when calculating the new $\alpha$-enhanced SSP model SEDs, and therefore the results shown here have the potential to be modified. Ideally, we should use stellar spectra of appropriate abundance ratios, and this is exactly the case since our library of empirical spectra is based on observations of local metal-poor stars which show the same abundance pattern (e.g., $\alpha$-element enhancement) as in Galactic GCs (see, e.g., Table 1 in Salaris \& Weiss 1998 and references therein). If there is any inconsistency between the spectral library and the stellar models used to determine the age of 47 Tuc, it is when considering models with scaledsolar abundances. However, in this case, we selected the required stellar spectra following their $[\mathrm{Fe} / \mathrm{H}]$ rather than their total metallicity $Z$. In this way, at least, the Fe lines estimates from the new model SEDs are more secure. On the other hand, the fact that the Balmer lines are being studied means that the opacity changes (according to the chosen abundance ratios) operate mostly in the pseudocon- tinua and may not have a significative impact on the results discussed here.

Figure 4 shows that the $\mathrm{H} \gamma_{\sigma<130}$ values measured on the spectra synthesized on the basis of $\alpha$-enhanced isochrones yield considerably younger ages than the scaled-solar ones. To understand this point, we recall that VA99 have clearly shown that for the higher spectral resolutions, the weakening of the $\mathrm{H} \gamma$ feature at $4340 \AA$ because of a higher metallicity (caused by the lower temperatures of TO stars owing to higher opacity of the stellar matter) is compensated by a deepening of the adjacent iron lines (mainly at the blue side of $\mathrm{H} \gamma$ ). The $\mathrm{H} \gamma_{\sigma<130}$ index definition takes into account this effect and thus is insensitive to metallicity variations. Using the $\alpha$-enhanced isochrones at a given $[\mathrm{Fe} / \mathrm{H}]$, the global metallicity is higher and therefore the temperatures of the stars slightly lower, causing a weakening of the $\mathrm{H} \gamma$ feature which is not compensated by any deepening of the adjacent iron lines. Therefore, this effect is very similar to the one produced by an age increase. Furthermore, we also have tested $\alpha$-enhanced isochrones with $[\mathrm{Fe} / \mathrm{H}]=-0.3$ $(Z=0.02)$, confirming that for a given $\alpha$-enhancement the $\mathrm{H} \gamma_{\sigma<130}$ index preserved its age-resolving power.
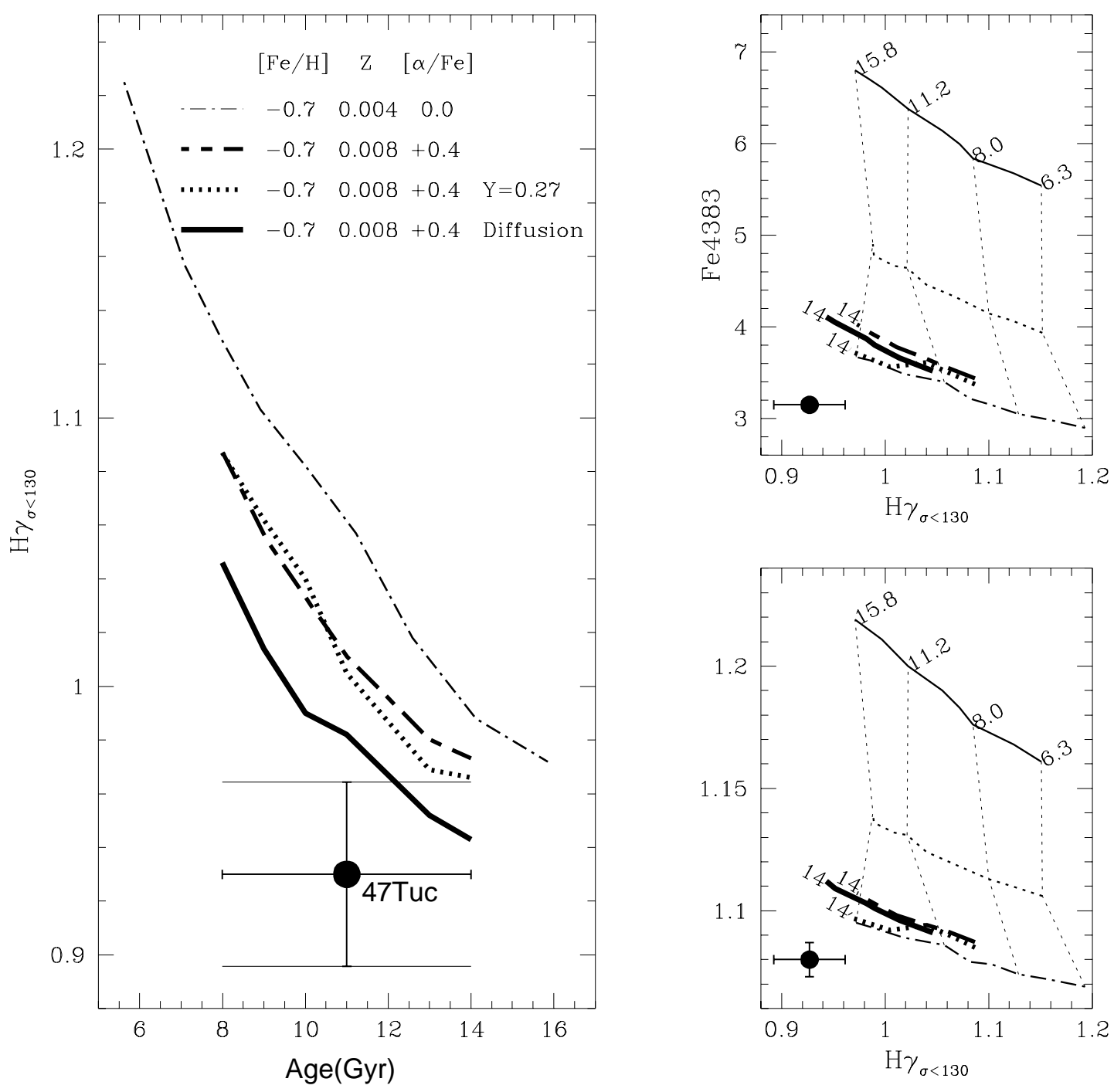

FIG. 4. - Left: $\mathrm{H} \gamma_{\sigma<130}$ measured on the integrated spectra synthesized on the basis of different isochrones. Right: Various metallicity indices are plotted vs. $\mathrm{H} \gamma_{\sigma<130}$. For the models represented by the thick lines, the ages vary from 8 to $14 \mathrm{Gyr}$. 


\subsection{Primordial Helium Content}

We find that a reasonable variation of the initial $\mathrm{He}$ content does not appreciably affect the spectroscopic age. In Figure 4 the $\mathrm{H} \gamma_{\sigma<130}$ values obtained on the basis of $\alpha$ enhanced isochrones with a solar initial $\mathrm{He}$ abundance $(Y=0.273)$ are not significantly different from the ones computed with $Y=0.254$ at the 47 Tuc metallicity $(\Delta Y /$ $\Delta Z=3)$.

\subsection{Atomic Diffusion}

Atomic diffusion is capable of changing the TO temperature of low-mass stars at a given age (see, e.g., Proffitt \& Vandenberg 1991; Chaboyer et al. 1992; Castellani et al. 1997; Cassisi et al. 1998; Salaris, Groenewegen, \& Weiss 2000). The occurrence of this physical process in the Sun has been demonstrated by helioseismic studies (e.g., Guenther et al. 1996). Lebreton et al. (1999) showed that diffusion is required to reproduce the temperatures of Hipparcos subdwarfs with $-1.0<[\mathrm{Fe} / \mathrm{H}]<-0.3$. Because of diffusion, the surface metallicity and He content decrease during the main-sequence (MS) phase due to their sinking below the convective envelope. Around the $\mathrm{TO}$, the surface $[\mathrm{Fe} / \mathrm{H}]$ and $Y$ show a minimum; then evolutionary timescales become much shorter, and diffusion is no longer effective. Moreover, since envelope convection deepens, almost all metals and $\mathrm{He}$ diffused toward the center are engulfed again in the convective envelope. Along the red giant branch the surface $[\mathrm{Fe} / \mathrm{H}]$ (and $\mathrm{He}$ ) is restored to nearly its initial value (the current spectroscopic determinations of $[\mathrm{Fe} / \mathrm{H}]$ for GCs make use of red giant stars, so that the measured metal abundances truly reflect the initial ones). For a given initial chemical composition and age, TO temperatures are significantly cooler than in isochrones without diffusion (the TO luminosities are reduced too, thus causing an age reduction by $\simeq 1 \mathrm{Gyr}$ if the TO brightness is used as an age indicator). The reason for this behavior is that the inward settling of
He during the MS raises the core molecular weight and the molecular weight gradient between surface and center of the star. This increases the stellar radius and the rate of energy generation in the center. The diffusion of the metals partially counterbalances this effect by decreasing the opacity in the envelope and increasing the central CNO abundance.

We have calculated a new set of isochrones (initial metallicity $[\mathrm{Fe} / \mathrm{H}]=-0.7)$ with $\mathrm{He}$ and metals diffusion included as in Salaris et al. (2000) and using the same input physics and $\alpha$-enhancement as in SW98. Figure 5 shows the fit to the 47 Tuc CMD of both Kaluzny et al. (1998) and Hesser et al. (1987) using these isochrones. The derived age (determined from the TO luminosity once the distance is fixed) is 9-11 Gyr. We obtain similar results when fitting the $V-I$ versus $V$ diagram using data of Kaluzny et al. (1998), with the exception that a better fit is attained for the giant branch than in the $B-V$ versus $V$ diagram.

Figure 4 shows the $\mathrm{H} \gamma_{\sigma<130}$ measurement for the model spectra computed on the basis of these new isochrones; the CMD age as estimated from Figure 5 is still smaller by $\sim 2$ Gyr than the minimum age allowed by the $\mathrm{H} \gamma_{\sigma<130}$ measurement but much more consistent with the $\mathrm{H} \gamma$ age derived employing the same set of isochrones than obtained before. The right panels of Figure 4 show that use of these new models does not significantly change our metallicity estimate for the cluster.

\section{CONCLUSIONS}

We have discussed the origin of the discrepancy between the spectroscopic (based on the effective temperature of TO stars) and CMD (based on the luminosity of TO stars and an assumed distance scale) age estimate for 47 Tuc as raised by G99. For this purpose we have defined a new age indicator, $\mathrm{H} \gamma_{\sigma<130}$, particularly suitable for studying GCs and low-velocity dispersion galaxies, which shows a superb power to break the age-metallicity degeneracy. $\mathrm{H} \gamma_{\sigma<130}$

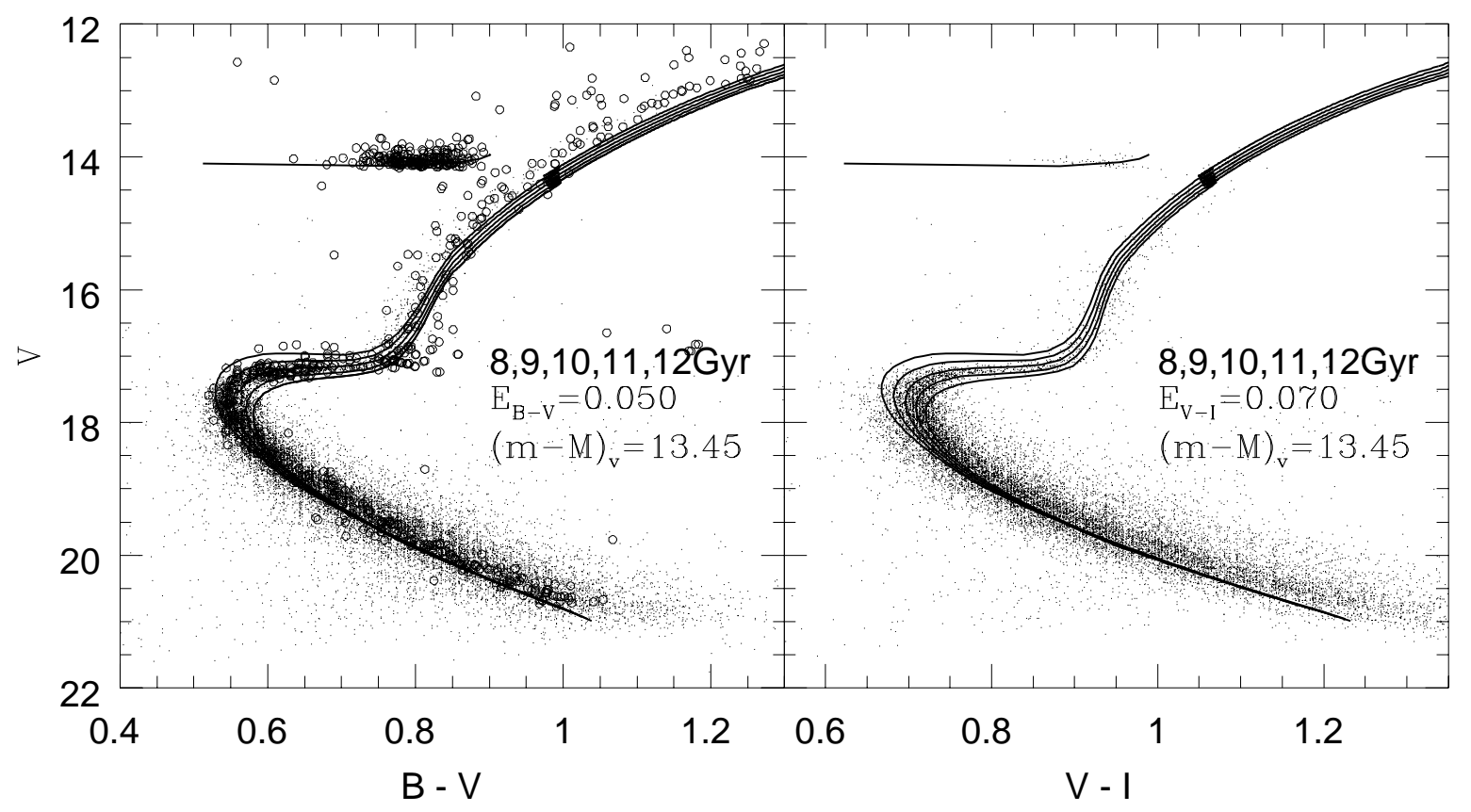

FIG. 5. - Left: We plot the CMD of 47 Tuc (data of Kaluzny et al. 1998 plotted as small circles; those of Hesser et al. 1987 plotted as large circles). Overplotted are various isochrones of $[\mathrm{Fe} / \mathrm{H}]=-0.7,[\alpha / \mathrm{Fe}]=+0.4, Z=0.008$, and atomic diffusion for different ages. Right: We plot the $V-I$ vs. $V$ diagram using data of Kaluzny et al. (1998). 
confirms the age discrepancy found by G99 for 47 Tuc. Emission fill-in of the Balmer lines appears to be an unlikely source of the weak $\mathrm{H} \gamma$ in 47 Tuc since the ages derived from different Balmer lines give discordant results if the hypothetical emission fill-in is corrected for and since a composite of four other metal-rich Galactic globular clusters shows the same weak $\mathrm{H} \gamma$ phenomenon. Thus, the fact that other metal-rich GCs show very similar low Balmer values in comparison to the model predictions suggests a problem in the zero point of current stellar population models. It is worth noting that this zero-point problem of the models with respect to the metal-rich GCs also works out for old elliptical galaxies.

We therefore analyzed the possible causes of the problem by studying a number of input parameters of the evolutionary computations and comparing the observed value of $\mathrm{H} \gamma_{\sigma<130}$ with that derived from the synthesized integrated spectra. Neither the initial He content nor the HB have significant effects on the Balmer indices synthesized for 47 Tuc. However, the inclusion of $\alpha$-element enhancement and atomic diffusion in the evolutionary models provide spectroscopic ages which are much closer to the CMD-derived ages. This occurrence constitutes a possible solution to the age discrepancy between CMD and integrated spectrum ages of old metal-rich stellar populations.

It is important to study if the age discrepancy is present in metal-poor GCs. For this purpose we need to expand the current stellar spectral libraries which feed the stellar population models (see V99) to extend their predictions to lower metallicities.

We are grateful to the nonanonymous referee G. Worthey for very helpful suggestions which helped to improve the final version of the paper. We thank S. Covino and L. Jones for providing us with spectra of 47 Tuc and M32, respectively. We also thank A. Bressan for a useful discussion. A. V. acknowledges the support of the PPARC rolling grant "Extragalactic Astronomy and Cosmology in Durham 1998-2002."
Alonso, A., Arribas, S., \& Martínez-Roger, C. 1999, A\&AS, 140, 261

Alonso, A., Salaris, M., Martínez-Roger, C., Straniero, O., \& Arribas, S. 1997, A\&A, 323, 374

Brown, J. A., \& Wallerstein, G. 1992, AJ, 104, 1818

Brown, J. A., Wallerstein, G., \& Oke, J. B. 1990, AJ, 100, 156

Burstein, D., Faber, S., Gaskell, C., \& Krumm, N. 1984, ApJ, 287, 586

Buzzoni, A., Mantegazza, L., \& Gariboldi, G. 1994, AJ, 107, 513

Carney, B. W. 1996, PASP, 108, 900

Carretta, E., \& Gratton, R. 1997, A\&AS, 121, 95

Carretta, E., Gratton, R. G., Clementini, G., \& Fusi Pecci, F. 2000, ApJ, 533,215

Cassisi, S., Castellani, V., degl'Innocenti, S., \& Weiss, A. 1998, A\&AS, 129, 267

Castellani, V., Ciacio, F., degl'Innocenti, S., \& Fiorentini, G. 1997, A\&A, 322,801

Chaboyer, B., Deliyannis, C. P., Demarque, P., Pinsonneault, M. H., \& Sarajedini, A. 1992, ApJ, 388, 372

Cohen, J. H., Blakeslee, J. P., \& Ryzhov, A. 1998, ApJ, 496, 808

Covino, S., Galletti, S., \& Pasinetti, L. E. 1995, A\&A, 303, 79

D’Antona, F., Caloi, V., \& Mazzitelli, I. 1997, ApJ, 477, 519

Freytag, B., \& Salaris, M. 1999, ApJ, 513, L49

Gibson, B., Madgwick, D., Jones, L. A., Da Costa, G., \& Norris, J. 1999, AJ, 118, 1268 (G99)

Girardi, L., Bressan, A., Bertelli, G., \& Chiosi, C. 2000, A\&AS, 141, 371

Gratton, R. G., Fusi Pecci, F., Carretta, E., Clementini, G., Corsi, C. E., \& Lattanzi, M. 1997, ApJ, 491, 749

Gratton, R. G., Quarta, M. L., \& Ortolani, S. 1986, A\&A, 169, 208

Gratton, R. G., \& Sneden, C. 1991, A\&A, 241, 501

\section{REFERENCES}

Guenther, D. B., Kim, Y.-C., \& Demarque, P. 1996, ApJ, 463, 382

Hesser, J. E., Harris, W. E., Vandenberg, D. A., Allwright, J. W. B., Shott, P., \& Stetson, P. B. 1987, PASP, 99, 739

Jones, L. A. 1999, Ph.D. thesis, Univ. North Carolina, Chapel Hill

Jones, L. A., \& Worthey, G. 1995, ApJ, 446, L31

Kaluzny, J., Wysocka, A., Stanek, K., \& Krzeminsky, W. 1998, Acta Astron., 48, 439

Lebreton, Y., Perrin, M.-N., Cayrel, R., Baglin, A., \& Fernandes, J. 1999, A\&A, 350, 587

Liu, W. M., \& Chaboyer, B. 2000, ApJ, 544, 818

Norris, J., \& Da Costa, G. S. 1995, ApJ, 447, 680

Osterbrock, D. E. 1989, Astrophysics of Gaseous Nebulae and Active Galactic Nuclei (Mill Valley: University Science Books), 84

Proffitt, C. R., \& Vandenberg, D. A. 1991, ApJS, 77, 473

Rose, J. A. 1994, AJ, 107, 206

Rose, J. A., \& Tripicco, M. J. 1986, AJ, 92, 610

Salaris, M., Groenewegen, M., \& Weiss, A. 2000, A\&A, 355, 299

Salaris, M., \& Weiss, A. 1998, A\&A, 335, 943 (SW98)

Tripicco, M. J. 1989, AJ, 97, 735

Vandenberg, D., Swenson, F., \& Alexander, D. 2000, ApJ, 532, 430

Vazdekis, A. 1999, ApJ, 513, 224 (V99)

Vazdekis, A., \& Arimoto, N. 1999, ApJ, 525, 144 (VA99)

Vazdekis, A., Casuso, E., Peletier, R. F., \& Beckman, J. E. 1996, ApJS, 106, 307

Worthey, G. 1994, ApJS, 95, 107

Worthey, G., Faber, S., González, J., \& Burstein, D. 1994, ApJS, 94, 687

Worthey, G., \& Ottaviani, D. L. 1997, ApJS, 111, 377 